\title{
Intrahepatic Cholestasis Following Abuse of Powdered Kratom (Mitragyna speciosa)
}

\author{
Friedrich G. Kapp • Hans H. Maurer • \\ Volker Auwärter • Martin Winkelmann • \\ Maren Hermanns-Clausen
}

Published online: 29 April 2011

(C) American College of Medical Toxicology 2011

\begin{abstract}
Introduction Kratom (Mitragyna speciosa) is a common medical plant in Thailand and is known to contain mitragynine as the main alkaloid. According to an increase in published reports and calls at German poison control centers, it has been used more frequently as a drug of abuse in the western hemisphere during the last couple of years. Despite this increase, reports of severe toxicity are rare within the literature.

Case report We describe a case of a young man who presented with jaundice and pruritus after intake of kratom for 2 weeks in the absence of any other causative agent. Alkaloids of M. speciosa were detected in the urine.

Conclusion While M. speciosa is gaining in popularity among illicit drug users, its adverse effects remain poorly understood. This is the first published case of intrahepatic cholestasis after kratom abuse.
\end{abstract}

F. G. Kapp $(\square) \cdot$ M. Hermanns-Clausen

Poisons Information Center VIZ-Freiburg,

Department of Pediatrics and Adolescent Medicine,

University Medical Center Freiburg,

Mathildenstrasse 1,

79106 Freiburg, Germany

e-mail: friedrich.kapp@uniklinik-freiburg.de

H. H. Maurer

Institute of Experimental and Clinical Pharmacology

and Toxicology, Saarland University,

Homburg, Germany

V. Auwärter

Institute of Forensic Medicine,

University Medical Center Freiburg,

Freiburg, Germany

M. Winkelmann

Division of Internal Medicine, Rechbergklinik Bretten,

Bretten, Germany

\author{
Abbreviations \\ ANA Antinuclear antibodies \\ AP Alkalic phosphatase \\ BMI Body mass index \\ GC-MS Gas chromatography-mass spectrometry \\ AST Aspartate aminotransferase \\ ALT Alanine aminotransferase \\ HT Serotonin \\ INR International normalized ratio \\ LC-MS Liquid chromatography-mass spectrometry \\ PTT Partial thromboplastin time \\ Ref. Reference value
}

\section{Introduction}

Mitragyna speciosa is an indigenous tree of Southeast Asia whose leaves have been utilized in Thailand and Malaysia over a long period of time as a drug to enhance the ability to work, as a narcotic, or as an alternative to opium [1,2]. M. speciosa is an illegal or controlled substance in several states (depending on the country either mitragynine, the plant itself, or its extracts are illegal), for example, Thailand, Australia, and Denmark [3, 4]. A marked increase of its abuse in the western hemisphere has been noted in the last couple of years [5], but reports of toxicity other than seizures or addiction are rare. We present the first case of intrahepatic cholestasis probably linked to a massive overdose of kratom.

\section{Case Description}

Our patient, a 25 -year-old man, with a BMI of $23.4 \mathrm{~kg} / \mathrm{m}^{2}$, ingested kratom for 2 weeks. He was aware of and curious 
about the effects of the drug and ordered the powdered substance via the Internet (www.thai-kratom.de), where it was advertised as an additive for footbaths. He ordered two kinds of powder (so-called Thai Pimp and Malaysian Green, both powder from ground up leaves) and took both powders on a daily basis orally, washing the powder down with orange juice. The ingested amount started with one to two teaspoons (one teaspoon is about 2.3-3.5 g, corresponding to approximately five to eight dried leaves) twice daily. In the course of 2 weeks, he increased the intake to four to six teaspoons daily. No further illicit drugs, medication (e.g., antibiotics, analgesics), dietary supplements, or alcohols were consumed; the patient's diet was adequate, and he was otherwise healthy. He described the effect of the drug as mildly relaxing, causing tiredness, and noticed a loss of appetite, without observing any stimulating effects. After cessation of drug intake due to inconvenient swallowing, he developed subjective fever and chills on day 2 after cessation, which lasted for about 1 week. On day 5 after cessation, he noticed slight abdominal discomfort, which developed into intense abdominal pain on day 8 and concomitant brown discoloration of the urine. The next day, the patient developed noticeable jaundice and pruritus and consulted his physician. He was admitted to hospital on that same day, approximately 10 days after cessation. The initial physical examination showed a pronounced jaundice but was otherwise unremarkable (blood pressure, 130/ $80 \mathrm{mmHg}$; heart rate, $72 / \mathrm{min}$; body temperature, $37.8^{\circ} \mathrm{C}$ ). In the initial laboratory tests, an elevated direct bilirubin of $28.6 \mathrm{mg} / \mathrm{dl}(489.1 \mu \mathrm{mol} / 1$; reference value (Ref.), $<0.3 \mathrm{mg} / \mathrm{dl}$ ) was found, while the indirect bilirubin was $2.3 \mathrm{mg} / \mathrm{dl}$ (39.3 $\mu \mathrm{mol} / \mathrm{l}$; Ref., $<0.9 \mathrm{mg} / \mathrm{dl}$ ). The alkalic phosphatase (AP) and the transaminases were only slightly elevated, AP being 173 U/1 (Ref., 40-130 U/1), AST 66 U/1 (Ref., $<50$ U/l), and ALT $94 \mathrm{U} / 1$ (Ref., $<50 \mathrm{U} / 1$ ), and did not rise above this level during the observation of the patient. Creatinine was $1.0 \mathrm{mg} / \mathrm{dl}(88.5 \mu \mathrm{mol} / \mathrm{l}$; Ref., $<1.2 \mathrm{mg} / \mathrm{dl})$, blood urea was $22 \mathrm{mg} / \mathrm{dl}$ (3.67 mmol/l; Ref., $<50 \mathrm{mg} / \mathrm{dl}$ ), INR was 1.15 (Ref., <1.15), and PTT was $34.5 \mathrm{~s}$ (Ref., 25.0-35.0 s). No signs of infection were observed clinically or in laboratory tests; viral hepatitis (hepatitis A, B and C) and autoimmune diseases (i.e., ANA) were ruled out serologically. Ultrasound and computed tomography of the abdomen showed signs of steatosis of the liver. Neither gallstones nor intra- or extrahepatic bile duct dilation was detected (common hepatic duct, $3 \mathrm{~mm}$ ). Upon further questioning, the patient reported the use of kratom but denied further drug abuse or intake of medication, including analgesic drugs and antibiotics even after repeated inquiry. The routine drug screen on admission had been negative (e.g., amphetamines, benzodiazepines, opioids tested), but alkaloids of $M$. speciosa were detected in the urine (see Table 1). To further elucidate the etiology of the intrahepatic cholestasis, a biopsy of the liver was performed on day 2 after admission. The pathologist identified a drug-induced cholestatic injury without hepatocellular damage (so-called pure cholestasis) with bile precipitations in hepatocytes and in fat vacuoles of single steatotic hepatocytes. The sinusoids were slightly distended and hyperemic, with evidence of modest inflammation indicating a diagnosis of canalicular cholestasis. The patient was neither a weight lifter nor did he take androgens or dietary supplements (a common trigger for the onset of cholestasis). Furthermore, the patient reported insomnia and restlessness which began shortly after the cessation of kratom powder intake, possibly as a sign of drug dependence and withdrawal, although the pruritus certainly contributed to these symptoms. The patient was then seen for follow-up in the clinic and showed slowly falling direct bilirubin levels (see Table 1), the pruritus also abated. The urine taken on day 47 after cessation was again analyzed for alkaloids of $M$. speciosa and their metabolites; no alkaloids could be detected at that time.

\section{Analysis}

Samples of the kratom powders used by the patient ("Thai Pimp" and "Malaysian Green") were analyzed by gas chromatography-mass spectrometry (GC-MS) to confirm the use of $M$. speciosa and to exclude other toxic ingredients. Therefore, $100 \mathrm{mg}$ of each powder was mixed with $1 \mathrm{ml}$ of ethanol, using a vortexer, and centrifuged. A $10-\mu l$ aliquot of the organic phase was transferred into a GC vial and dried under a stream of nitrogen. After dissolving the extract in $100 \mu \mathrm{l}$ ethyl acetate, $1 \mu \mathrm{l}$ was injected into a GC-MS system (6890 Series GC combined with a 5973 Series mass selective detector and ChemStation G1701GA, D.03.00.611, Agilent Technologies, Waldbronn, Germany) equipped with a HP-5ms capillary column $(30 \mathrm{~m} \times 0.25 \mathrm{~mm}$ I.D., $0.25 \mu \mathrm{m}$ film thickness from $\mathrm{J} \& \mathrm{~W}$ Scientific, Folsom, CA, USA). The MS was operated in full scan mode, and the received spectra were compared to
Table 1 Laboratory tests during the course of treatment, days after cessation

$* 1 \mathrm{mg} / \mathrm{dl}=17.1 \mu \mathrm{mol} / \mathrm{l}$

\begin{tabular}{lcccccc}
\hline & Day 12 & Day 14 & Day 16 & Day 25 & Day 35 & Day 47 \\
\hline Direct bilirubin (mg/dl)* & 28.6 & 26.7 & 28.6 & 29.3 & 24.7 & 5.8 \\
Urine mitragynine & & Positive & & & & Negative \\
Serum mitragynine (ng/l) & 20 & 20 & & & \\
\hline
\end{tabular}


common MS libraries for identification of the compounds. Through detection of mitragynine (both samples) and traces of rhynchophylline (only in "Thai Pimp"), besides typical phytosterols, the samples were identified as M. speciosa. Although mitragynine was not quantified, the analysis revealed that the sample of "Thai Pimp" contained significantly more mitragynine than the sample of "Malaysian Green." In both samples, no synthetic adulterants or contaminations were detected.

Both urine and serum samples were analyzed for the main alkaloids of M. speciosa and their metabolites by Liquid chromatography-MS with a linear ion trap according to the publications of Philipp et al. [6, 7]. Briefly, mitragynine and its metabolites could be detected in urine after solid-phase extraction, LC separation, and comparison of the product ion mass spectra with the reference spectra published by Philipp et al. [7]. In the serum sample, the main kratom alkaloid was semi-quantified using one-point calibration with mitragynine reference standard. The results are summarized in Table 1. In all samples, paynantheine and its metabolites could not be detected, most probably due to its lower content in the kratom preparation used and the rather long time interval since application.

\section{Discussion}

M. speciosa is a plant of Southeast Asian origin. It has been used as a drug for a long time in this area, especially in Thailand and Malaysia, due to its opioid-like effects. Major constituents (among many others) of young leaves of Thai M. speciosa are mitragynine (66\%), paynantheine $(9 \%)$, speciogynine $(7 \%)$, 7-hydroxymitragynine $(2 \%)$, and speciociliatine $(1 \%)$ in which the relative amount varies greatly according to the origin of the plant. Corynantheidine was discovered in the extract of the leaves, but the relative content was not specified [8]. Mitragynine, the main alkaloid of over 20 known alkaloids in M. speciosa, has been fully characterized in 1964 [9]. Of the abovementioned alkaloids, mitragynine, corynantheidine, and 7hydroxamitragynine are known to be pharmacologically active, but the other constituents might also provide a pharmacological effect. The opioid-like effects of analgesia [8] and reduced gastrointestinal motility $[1,10]$ are attributed to mitragynine, which acts at $\mu$ - and $\delta$-opioid receptors [8]. 7-Hydroxymitragynine shows a potent analgesic effect in rats even superior to morphine [11] and acts mainly at $\mu$-opioid receptors and to a lesser extent at $\mathrm{K}$ opioid receptors. Corynantheidine also binds at $\mu$-receptors but acts as a functional antagonist since it does not activate this receptor [8]. In addition to the opioid receptor-mediated effects, there are conflicting data on the effect of mitragynine on $\alpha_{2}$ and $\mathrm{HT}_{2 \mathrm{~A}}$ receptors [12-14]. Kratom is often said to have coca-like effects (first described in 1932 [15]), but except the increased ability to work, there is little evidence for amphetamine-like effects and the possible pharmacological mechanisms remain unclear. The leaves of $M$. speciosa are usually chewed fresh, though alternatives include eating dried leaves ground to powder, smoking (e.g., dried leaves), drinking teas, or using kratom resin [2]. The dosage in most kratom users varies between 10 and 30 chewed leaves per day [16]. The total yield of crude alkaloid in M. speciosa seems to be about $0.25 \%$ [17], tested in plants of Thai origin. A fresh leaf of $M$. speciosa weighs approximately $1.7 \mathrm{~g}$,

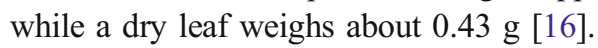

The lack of any other known causative agents might suggest the powdered M. speciosa as a probable [18] underlying factor for the intrahepatic cholestasis. This was an unexpected finding as there have been few published reports describing toxicity of kratom, although an increase in kratom use has been observed over the last couple of years [5]. This increase is probably in part due to the wide and rapidly growing availability on the Internet and its discussion in drug-related forums $[4,19]$ as well as to a general hype about "legal highs," which significantly increased after "Spice" and similar products gained high popularity starting in 2008 [20, 21]. Three reports of seizures after kratom use have been identified. In two of these cases, the drug was used for pain management [22] and to counter symptoms of opioid withdrawal [23]. At least one additional drug (Datura [22], modafinil [23]) was used at the onset of seizures, followed by coma in one case report [22]. In the third case of seizures [24], kratom was used as a recreational drug and additional drugs were not reported. To date, there have been no reports of hepatic toxicity of $M$. speciosa published in the literature.

To our surprise, and despite the long latency between cessation of kratom and admission to the hospital, mitragynine and its metabolites were detectable in the urine sample taken approximately 2 weeks after cessation of drug use, proving the intake of the drug. While it cannot be excluded that the patient might have been inaccurate concerning the time of cessation, it is highly unlikely that he took kratom during his hospital stay. Thus, the shortest latency could be 2-3 days between the last kratom use and the first sampling of urine (on day 3 after admission to the hospital), though was more likely to be 10-14 days. So far, the elimination half-life (serum and plasma) was only studied for mitragynine in rats and was found to be approximately $4-9 \mathrm{~h}$ after a single dose $[25,26]$. Considering the described half-life, the finding of mitragynine in the serum and in the urine of the patient after the assumed interval suggests a severely prolonged elimination half-time of the alkaloids. It must be kept in mind though that the toxicokinetics in humans, of alkaloids of $M$. speciosa other than mitragynine or after repeated ingestion, have not been 
investigated yet. Nonetheless, a prolonged elimination halflife of mitragynine seems to be probable and might be due to a number of reasons (e.g., saturation of enzymatic pathways, sustained release out of an internal reservoir after initial distribution into tissues). While this remains speculative, prolonged elimination half-lives have been observed after severe overdose (Hans H. Maurer, personal communication, 2010). As was shown by Philipp et al., mitragynine and paynantheine are metabolized in rat and humans liver via phases I (hydrolysis and dealkylation) and II (conjugation to glucuronides and sulfates) reactions [6, 7]. Due to the extensive liver metabolism of mitragynine and other alkaloids of M. speciosa, hepatic toxicity (e.g., in form of intrahepatic cholestasis as observed in the patient) seems plausible and might have been amplified by the preexisting liver damage (steatosis hepatis). The prolonged half-live of the alkaloids could either be secondary to delayed hepatic clearance due to the hepatic injury and/or to distribution into tissues (e.g., the liver). Both hypotheses might explain why mitragynine was still detectable in the urine after 2 weeks. Prolongation of elimination half-life has been described after overdoses of therapeutic drugs [27, 28]. Unfortunately, the liver biopsy was too small to be analyzed for its content of M. speciosa alkaloids. Clearly, future research is needed to confirm if alkaloids and other compounds of M. speciosa accumulate in the liver.

Why the patient developed intrahepatic cholestasis remains unclear, but the massive overdose of up to six teaspoons (about 14-21 g, approximately 32-49 dried leaves; usual dosage, 10-30 leaves) daily for 2 weeks may have been an etiologic factor. Further substances present in M. speciosa apart from the main alkaloids could also trigger toxic effects and might only be toxic when the usual amount of intake is significantly exceeded. The effect of recently published substances in M. speciosa (alkaloids, flavonoids, saponins, triterpenoid saponins, and glycoside derivatives) [29] on health and, in particular, on the liver are not studied well. A recent study showed a slight elevation in glutathione- $S$-transferase in mice after administration of M. speciosa extracts [30] as a possible sign of strain on the liver. Other than that, toxicity studies are rare and do not show relevant toxicity in most reports. Exceptions are the publications by Grewal, who observed seizures at very low doses [15], and by Reanmongkol et al., who observed convulsions at very high doses [31]. The same dosages did not result in toxicity in the experiments of other groups $[1,29]$. Interestingly, a discoloration of the skin and a hepatic face have been described in the literature $[2,15,16]$.
This case description is the first report of intrahepatic cholestasis after kratom abuse with possibly a prolonged elimination half-life. It must be kept in mind though that the patient's history might have been inaccurate concerning the time of the last intake of kratom, especially when he experimented with a drug, which he bought on the Internet. As is often the case, the intake of medication or other drugs cannot be ruled out with certainty, with the patient possibly concealing this or the long interval between intake and presentation at the hospital, making the detection in drug screens impossible. The possible link between kratom and intrahepatic cholestasis as a form of hepatic toxicity remains intriguing and encourages more research on the subject of $M$. speciosa, its metabolism in humans, and its adverse effects.

\section{Conclusion}

Despite a better characterization of its main alkaloids and their respective functions, the adverse effects of kratom use remain poorly understood. We report the first case of probable hepatic toxicity in the form of intrahepatic cholestasis and see a need for further evaluation of kratom, its pharmacology, and its adverse effects.

\section{References}

1. Chittrakarn S, Sawangjaroen K, Prasettho S, Janchawee B, Keawpradub N (2008) Inhibitory effects of kratom leaf extract (Mitragyna speciosa Korth.) on the rat gastrointestinal tract. J Ethnopharmacol 116(1):173-178

2. Jansen KL, Prast CJ (1988) Ethnopharmacology of kratom and the mitragyna alkaloids. J Ethnopharmacol 23(1):115-119

3. Narcotics Act B.E. 2552 (1979). http://www.aseansec.org/Narcotics $\% 20$ Act $\% 20$ B.E. $\% 202552 \% 20(1979) \% 20-\% 20$ Thailand. doc. Accessed 10 Jan 2011

4. Erowid kratom (Mitragyna speciosa) vault (2010) (http://www. erowid.org/plants/kratom/kratom.shtml). Accessed 12 Dec 2010

5. Babu KM, McCurdy CR, Boyer EW (2008) Opioid receptors and legal highs: Salvia divinorum and kratom. Clin Toxicol 46 (2):146-152

6. Philipp AA, Wissenbach DK, Zoerntlein SW, Klein ON, Kanogsunthornrat J, Maurer HH (2009) Studies on the metabolism of mitragynine, the main alkaloid of the herbal drug kratom, in rat and human urine using liquid chromatography-linear ion trap mass spectrometry. J Mass Spectrom 44(8):1249-1261

7. Philipp AA, Wissenbach DK, Weber AA, Zapp J, Zoerntlein SW, Kanogsunthornrat J, Maurer HH (2010) Use of liquid chromatography coupled to low- and high-resolution linear ion trap mass spectrometry for studying the metabolism of paynantheine, an alkaloid of the herbal drug kratom in rat and human urine. Anal Bioanal Chem 396(7):2379-2391 
8. Takayama H (2004) Chemistry and pharmacology of analgesic indole alkaloids from the rubiaceous plant, Mitragyna speciosa. Chem Pharm Bull 52(8):916-928

9. Shellard EJ (1974) The alkaloids of Mitragyna with special reference to those of Mitragyna speciosa, Korth. Bull Narc 26 (2):41-55

10. Watanabe K, Yano S, Horie S, Yamamoto LT (1997) Inhibitory effect of mitragynine, an alkaloid with analgesic effect from Thai medicinal plant Mitragyna speciosa, on electrically stimulated contraction of isolated guinea-pig ileum through the opioid receptor. Life Sci 60(12):933-942

11. Matsumoto K, Horie S, Ishikawa $H$, Takayama $H$, Aimi N, Ponglux D, Watanabe K (2004) Antinociceptive effect of 7hydroxymitragynine in mice: discovery of an orally active opioid analgesic from the Thai medicinal herb Mitragyna speciosa. Life Sci 74(17):2143-2155

12. Matsumoto K, Mizowaki M, Suchitra T, Murakami Y, Takayama H, Sakai S, Aimi N, Watanabe H (1996) Central antinociceptive effects of mitragynine in mice: contribution of descending noradrenergic and serotonergic systems. Eur J Pharmacol 317(1):75-81

13. Matsumoto K, Mizowaki M, Takayama H, Sakai S, Aimi N, Watanabe H (1997) Suppressive effect of mitragynine on the 5methoxy- $N, N$-dimethyltryptamine-induced head-twitch response in mice. Pharmacol Biochem Behav 57(1-2):319-323

14. Tohda M, Thongpraditchote S, Matsumoto K, Murakami Y, Sakai S, Aimi N, Takayama H, Tongroach P, Watanabe H (1997) Effects of mitragynine on cAMP formation mediated by delta-opiate receptors in NG108-15 cells. Biol Pharm Bull 20(4):338-340

15. Grewal KS (1932) Observations on the pharmacology of mitragynine. J Pharmacol Exp Ther 46(3):251-271

16. Suwanlert S (1975) A study of kratom eaters in Thailand. Bull Narc 27(3):21-27

17. Chittrakarn S, Keawpradub N, Sawangjaroen K, Kansenalak S, Janchawee B (2010) The neuromuscular blockade produced by pure alkaloid, mitragynine and methanol extract of kratom leaves (Mitragyna speciosa Korth.). J Ethnopharmacol 129(3):344-349

18. UMC (2010) (http://www.who-umc.org/DynPage.aspx? id=22682). Accessed 10 Mar 2011

19. NDIC (2005) Herbal drug update: kratom. Narcotics Digest Weekly 4(16):4

20. Auwärter V, Dresen S, Weinmann W, Müller M, Pütz M, Ferreirós N (2009) 'Spice' and other herbal blends: harmless incense or cannabinoid designer drugs? J Mass Spectrom 44(5):832-837
21. EMCDDA (2009) Understanding the 'Spice' phenomenon. European Monitoring Centre for Drugs and Drug Addiction, Lisbon

22. Nelsen JL, Lapoint J, Hodgman MJ, Aldous KM (2010) Seizure and coma following kratom (Mitragynina speciosa Korth) exposure. J Med Toxicol 6(4):424-426

23. Boyer EW, Babu KM, Adkins JE, McCurdy CR, Halpern JH (2008) Self-treatment of opioid withdrawal using kratom (Mitragynia speciosa korth). Addiction 103(6):1048-1050

24. Roche KM, Hart K, Sangalli B, Lefberg J, Bayer M (2009) Kratom: a case of a legal high; Abstracts of the 2008 North American Congress of Clinical Toxicology Annual Meeting, September 11-16, 2008, Toronto, Canada. Clin Tox 46(7), p. 598

25. de Moraes NV, Moretti RAC, Furr EB, McCurdy CR, Lanchote VL (2009) Determination of mitragynine in rat plasma by LC-MS/ MS: application to pharmacokinetics. J Chromatogr B Analyt Technol Biomed Life Sci 877(24):2593-2597

26. Janchawee B, Keawpradub N, Chittrakarn S, Prasettho S, Wararatananurak P, Sawangjareon K (2007) A high-performance liquid chromatographic method for determination of mitragynine in serum and its application to a pharmacokinetic study in rats. Biomed Chromatogr 21(2):176-183

27. Jimmink A, Caminada K, Hunfeld NGM, Touw DJ (2008) Clinical toxicology of citalopram after acute intoxication with the sole drug or in combination with other drugs: overview of 26 cases. Ther Drug Monit 30(3):365-371

28. Bodmer M, Burkard T, Kummer O, Beyrau R, Krähenbühl S, Haschke M (2008) Pharmacokinetics and pharmacodynamics of quetiapine in a patient with a massive overdose. Ther Drug Monit 30(4):553-556

29. León F, Habib E, Adkins JE, Furr EB, McCurdy CR, Cutler SJ (2009) Phytochemical characterization of the leaves of Mitragyna speciosa grown in U.S.A. Nat Prod Commun 4(7):907-910

30. Azizi J, Ismail S, Mordi MN, Ramanathan S, Said MIM, Mansor SM (2010) In vitro and in vivo effects of three different mitragyna speciosa korth leaf extracts on phase II drug metabolizing enzymes-glutathione transferases (GSTS). Molecules 15 (1):432-441

31. Reanmongkol W, Keawpradub N, Sawangjaroen K (2007) Effects of the extracts from Mitragyna speciosa Korth. leaves on analgesic and behavioral activities in experimental animals. Songklanakarin J Sci Techno 29(suppl 1):39-48 